\title{
Comparison of Chinese and American Sign Language Interpretation Majors and Some Enlightenment
}

\author{
Ting Yang \\ School of Foreign Language Studies \\ Shaanxi University of Chinese Medicine \\ Xianyang, China
}

\begin{abstract}
To cultivate better sign language interpreters and improve the Chinese sign language interpretation profession, the paper compares the curriculum design, test system, certification system and employment situation of Chinese and American sign language interpretation majors. The results suggest that more Chinese universities should set up sign language interpretation major in the undergraduate period, the curriculum of sign language interpretation specialty in China should increase interpretation related courses, and the government should provide special funds for the training and employment of sign language interpreters.
\end{abstract}

Keywords-Sign Language Interpretation; China; U.S.; Comparison; Enlightenment

\section{INTRODUCTION}

On January 1, 2007, the Ministry of Labor and Social Security held a new career information conference in Shanghai, officially releasing 10 new occupations to the public, one of which was the sign language interpreter. "Sign Language" has long been considered an imperfect and complex "communication system" until 1960, when William Stokoe, the father of American sign language, published his book Sign Language Structure: An Outline of the Visual Communication Systems of the American Deaf, sign language was recognized and accepted as a natural language for human beings. The "sign language interpreter" is defined as a group that provides interpretation services for the hearing impaired by the transformation between sign language and spoken language[1].

Since the establishment of the US "Registry of Interpreters for the Deaf(RID)" in 1964, sign language interpretation has gone through 50 years of history. The United States is a pioneer in this field, and other countries have subsequently filled the gap in this field[2]. However, in China, the study of sign language interpretation started very late, and the first article describing sign language interpretation appeared in 2001 in China National Knowledge Internet(CNKI).

In fact, there are at least 20.57 million hearing-impaired people in China, and the number of sign language interpreters who can provide services is very small and cannot meet the needs of this special group. The mechanism for cultivating sign language interpreters is not comprehensive enough. Therefore, it is necessary to carry out comparisons in various aspects, and learn from the practice of the pioneer in the United States in this field.

\section{CurRiculum Design}

At present, only Zhongzhou University, Nanjing vocational and Technical College of special education and Liaoning Yingkou vocational and technical college have set up sign language interpretation major in China, which are listed as specialized vocational education, some are called sign language interpretation major, some are called special education major It can be seen that the naming is not uniform.

In addition, taking one of the universities as an example, the training goal is to make students proficient in the use of Chinese deaf natural sign language and the current Chinese universal sign language and to freely interpret Chinese and sign language. However, its curriculum is Chinese Sign Language, Deaf Natural Sign Language, Sign Language Reading and Using Practice, Professional Sign Language, Introduction to Sign Language Linguistics, Introduction to International Sign Language, Deaf and Society, History of Deaf Education, Psychology and Education of Deaf Children, Introduction to Special Education, Introduction to Sign Language Interpretation, Modern Chinese, etc. It is not hard to see that the emphasis of the course design is still on the study of sign language, but not the conversion between sign language and Chinese, namely, lacking the course of sign language interpretation or interpretation-related course. Although there are some sign language training institutions in China, their curriculum design also attaches great importance to sign language learning rather than interpretation ability. Taking Beijing Feiyu Sign Language Interpretation Training Course as an example, the training content is mainly about vocabulary in life, instead of interpretation strategies or methods.

From this point of view, although there are learning courses for sign language interpretation in China, the essence is equivalent to the learning of sign language, rather than the conversion between two languages.

In the United States, sign language interpretation major is widely found in more than 150 colleges and universities, of which more than 100 universities have set it up as a professional college education, more than 30 universities have

The paper is sponsored by Xianyang Science and Technology

Research Project 2017k02-103 
listed it as an undergraduate major, and the rest have put it in the graduate stage for students to study[3].

Taking the National Technical Institute for the Deaf of Rochester Institute of Technology as an example, its teaching objective is to help students master American Sign Language and sign language interpretation skills, finally pass the national interpreter certification (NIC) exam set by the sign language interpretation registration office, and use it as a skill to eventually find the right job. Its courses include American sign language, deaf expression, introduction to interpretation, Interpretation from English to American sign language, interpretation from American sign language to English, Sign Language Interpretation of applied and literary texts, educational Sign Language Interpretation, medical Sign Language Interpretation, legal Sign Language Interpretation, etc. Undergraduate courses include American Sign Language, Linguistics Foundation, Deaf Culture, Interpretation Standards, Interpretation Foundation, Conference Sign Language Interpretation, Interview Sign Language Interpretation, etc. In addition to the above courses, the postgraduate courses also require a sign language interpretation internship in the deaf community, which is a higher level than the undergraduate course.

It can be seen from the comparison that China has a single level of curriculum design and lacks interpretation related courses. Improving these two points should be a future concern.

\section{TEST System}

The test systems for sign language interpretation ability in Chinese mainland and Taiwan are different. This article takes Chinese mainland as an example. The test is held 4 times a year, including both written test and oral / sign language test. As long as the total score of the candidate is not less than 75, or the single subject is not less than 30 , the sign language employment ability qualification certificate can be obtained[4].

The written test is divided into two parts: non-video questions and video questions. Non-video questions require candidates to complete 40 fill-in-the-blank questions in half an hour, 10 related to sign language theories, 20 basic sign language symbols, and for each remaining 10, candidates are required to select the corresponding expression for the sign language shown in the picture from the given options. This part accounts for 25 points. The video questions give 5 situational dialogues. After each dialogue, there are 5 questions respectively. Candidates are required to select the correct answers to describe the scene in the $\mathrm{A}$ and $\mathrm{B}$ options according to the dialogue. Each dialogue will be played once. This part also takes up 25 points. The total score of the written examination is 50 .

The oral / sign language test lasts about 10 minutes and is also divided into two parts: the conversion of text to sign language and the communication as a mock sign language interpreter to help a deaf and hearing person. The second is an immersive live demonstration, because the deaf will only use sign language in the process, and the hearing person will only speak Chinese. The full score is also 50 points.

In the United States, two institutions are specially responsible for the issuance of sign language interpretation certificates, namely, the Registry of Interpreters for the Deaf (RID) and the National Association of the deaf (NAD). That is to say, sign language learners can choose any institution to test their sign language interpretation ability and obtain corresponding certificates. In fact, the two organizations jointly set up the National Council on Interpreting, which is responsible for the NAD-RID National Interpreter Certification (NIC). The test also consists of two parts: written test and oral / sign language test.

The written test consists of 150 multiple-choice questions covering five major areas: Society and culture, language history, social and political background of interpretation, sign language interpretation and employment of sign language interpreters. Candidates must answer at least 98 questions correctly to pass the test. Only after passing the written test can one enter the later test, namely the interpretation/sign language test. The part consists of three sections: the conversion of English to American sign language, the conversion of American sign language to English, and the two-way conversion of English and American sign language (through the live simulation of sign language interpreter to help the dialogue between deaf and hearing people). The entire process will be recorded in video form so that the scorer can give the corresponding score.

From this, both China and the United States have their own strengths. For example, in the written test in the United States, the scope of the test is broader, including society and culture, as well as the social and political background of interpretation. While in China, the oral/sign language test not only examines the bilingual conversion under the input of the sound symbol and sign language, but also the conversion process of the text form to the sign language.

\section{CERTIFICATION SYSTEM AND EMPLOYMENT}

In China, the only relevant information about the qualification of sign language interpreters is the National Vocational Standard, Sign Language Interpreters (Trial), which was piloted in 2009. The book clearly divides sign language interpreters into different levels: Level 2, Level 3, Level 4, and Level 5. Among them, the requirements of the second level are the highest: the practitioners must work in the industry for 8 years after obtaining the qualification certificate of the third-level sign language translator; or work in the industry for 5 consecutive years after obtaining the level 3 sign language interpreter qualification certificate, and obtain the completion certificate after learning and passing the level 2 sign language interpreter course; and level 5 sign language interpreter qualification certificate requires practitioners to work in the industry continuously for two years.

Although the level of sign language interpreters is clearly divided in this book, the employment situation of sign language interpreters in China is still not optimistic. Through the author's conversation with the teacher of Xi'an No.2 School for the Deaf and Dumb, most of the people engaged in sign language translation in Shaanxi Province are teachers of the School for the Deaf, rather than sign language interpreters. At present, the number of sign language interpreters is very small, which can't meet the needs of a large number of deaf people. In addition, three colleges and universities offering sign language 
interpretation major have an annual enrollment of about 150 (after 2011), among which Zhongzhou University began enrolling students in 2004, and Nanjing Special Education Vocational and Technical College began in 2005, while Yingkou Vocational and Technical College of Liaoning Province only starts in 2011. Based on the calculation of 150 students every year since 2004, there should be at least 2250 graduates. However, it is rarely seen that there is a job described as "sign language interpretation" in social recruitment positions, which shows that many graduates do not engage in this major after graduation.it is often the case that the teacher of the deaf-mute school will undertake the work instead of setting up a sign language interpretation post. In various public places, such as banks, hospitals, etc., hearing impaired people need sign language interpreters to help convey information, but there is no special sign language interpreter[5]. They are not in the position where there should be a sign language interpreter, so, their employment cannot be guaranteed, not to mention the issue of remuneration that has never been standardized in the market.

By comparison, the United States is more comprehensive than China in terms of qualification and employment. NIC is jointly issued by NAD and RID, which is divided into three levels. When the candidate passes the written test, and passes the score range in the one-way conversion between the two languages and the two-way scene conversion, he/she will be issued "sign language interpreter" certification. When the examinee passes the written test and gets the score within the passing range through the one-way conversion between the two languages respectively, and gets the high score in the two-way conversion of the scene, he/she will be issued the "senior sign language interpreter" certification. When the candidate passes the written test, and the scores of the latter parts are all high, he/she will be certified by the method of "expert sign language interpreter".

It is worth mentioning that in the United States, the system is eligible for review, that is, the certificate obtained above is valid within a certain time limit, and during this period, the licensee must collect information to prove that he has been practicing the skill or engaged in the industry. For example, the "Sign Language Interpreter" certificate is valid for 5 years. Therefore, the certificate holder must submit the required materials every 5 years, and the issuing agency will rate the contents of the materials to see whether they are qualified to continue to hold the certificate.

Under such a strict system, sign language interpreters have a variety of work options: school, medical and business fields. Taking Rochester Institute of Technology as an example, 122 full-time sign language interpreters were assigned to 121 majors to provide classroom translation services for deaf students. According to the US Bureau of Labor Statistics, at present, the average annual income of sign language interpreters in the United States is 45700 U.S. dollars (about 280000 Yuan). The places where sign language interpreters work most are primary schools, high schools, and government agencies, while the government agencies pay the highest wage to sign language interpreters, with an annual payment of 72330 U.S. dollars (about 440000 Yuan). The data also shows that as of May 2011, the average annual salary of sign language interpreters in various industries was about 51,000 US dollars (about 310,000 Yuan). In the field of science and technology, the electronics industry, sign language interpreters are paid equally well. The Bureau of Labor Statistics also predicts that the number of sign language interpreters will increase by $42 \%$ between 2010 and 2020, and a new demand: the remote sign language interpretation service has given birth to a new type of work, which is also about to become a new direction of talent cultivation in American universities in the future[6].

Obviously, the Chinese sign language interpreter's certification system is far less perfect than the United States, and the employment situation is not as good as the United States.

\section{SUMMARY}

First of all, there are obvious differences in curriculum design between the two countries. The curriculum design of Chinese colleges and universities is often based on sign language learning, while there are various interpretation related theories, skills or practice courses in American colleges and universities. Therefore, the curriculum of sign language interpretation specialty in China should increase interpretation related courses, so that students can practice more conversion between two languages.

Secondly, the education level of sign language interpretation major in China is too low to teach students in a comprehensive and systematic way under limited resources. Therefore, it should be considered to set up the major in the undergraduate stage as an extension of vocational education, and at the same time, it can cultivate higher-level talents.

Finally, the government should try to provide sign language interpreters with corresponding jobs and encourage them to various public places to help the hearing impaired. On the basis of the overall market, wages or minimum wages should be set up to protect the life of sign language interpreters.

It is not difficult to draw from the comparison of sign language interpretation major between the two countries: Only by perfecting the curriculum and improving the level of education, can a qualified sign language interpreter be trained. When qualified interpreters enter the market, the government should guarantee their employment by setting jobs or regulating the salary standard. In this way, sign language interpreters can be retained to help more hearing-impaired people and further ensure social harmony.

\section{ACKNOWLEDGMENT}

First and foremost, I appreciate my university which gives me a comfortable working atmosphere. Second, I would like to show my deepest gratitude to my husband, Bo Chen, who has walked me through all the stages of the writing of this thesis. Without his help, this thesis could not have reached its present form. My sincere appreciation also goes to my parents, who always supported me.

\section{REFERENCES}

[1] Kellett Bidoli, C.J, "Spoken-language and signed-language interpretation: Are they really so different ?,” in Interpreting in the 21st 
[4] Meng Fanling, "The social role and problems of news sign language interpretation,” in Language Research, 2001, pp. 87-88 (In Chinese)

century: Challenges and Opportunities: Selected Papers from the 1st For Conference on Interpreting Studies, G. Giuliana and M. Viezzi Eds. Amsterdam / Philadelphia: Academic, 2012, pp. 171-179.

[2] Miriam Shlesinger, "Signed-language interpreting as a fundamental human right," Bar lian Univesity Press, 2012, pp. 350-359.

[3] Kellett Bidoli, C.J, "Sign language: A newcomer to the interpreting forum,” The Interpreter's Newsletter, 2011, pp. 131-151
[5] Meng Fanling, "The occupational profile and social role of sign language interpreters,” in News Lover, 2008, pp.58-59 (In Chinese)

[6] Wang Jihong, "Research on sign language interpretation at home and abroad: history and current situation," in Shanghai Journal of Translators and Interpreters, 2009, pp. 23-28 (In Chinese) 